\title{
Energy spectrum of the organic quasi-1D conductors with NNN and correlated hopping
}

\author{
Yu.Skorenkyy, O.Kramar \\ Ternopil State Technical University, Department of Physics, \\ 56 Rus'ka Str., Ternopil UA-49001, Ukraine; Tel.:+380352251946, \\ Fax: +380352254983; E-mail: skorenky@tu.edu.te.ua
}

Received August 3, 2005, in final form February 27, 2006

\begin{abstract}
A model for organic quasi-one-dimensional conductors (TMTTF) ${ }_{2} \mathrm{X}$ and (TMTSF) ${ }_{2} \mathrm{X}$ is considered. The anisotropic character of these compounds is modelled by two different hopping parameters: $t$ between nearest neighbors (NN) in a chain of tetramethyl-tetrathiafulvalene (TMTTF) or tetramethyl-tetraselenfulvalene (TMTSF) molecules and $t^{\prime}$ between the chains (NNN - between next nearest neighbors). Taking into account the correlated hopping of electrons allows us to describe the effect of site occupancy on hopping processes. In a regime of strong intraatomic correlation, high energy processes are cut off by applying two successive canonical transformations. An effective model is obtained for concentration of electrons $n<1$ which contains kinetic exchange terms of antiferromagnetic (AF) nature. Oppositely, NNN hopping and correlated hopping disfavor the AF order. The energy spectrum of the effective model is calculated. Application of the obtained results to quasi-one-dimensional conductors is discussed.
\end{abstract}

Key words: quasi-one-dimensional organic conductors, next-nearest-neighbors hopping, correlated hopping, indirect exchange interaction

PACS: $74.25 .-q, 71.10 . F d$

\section{Introduction}

The diversity of organic compounds synthesized so far opens miraculous prospects for science and industry. Half a century ago it was not believed that organic compounds were capable of showing electrical and magnetic properties similar to transition metal compounds. Investigations of organic conductors were started about fifty years ago $[1,2]$ and were greatly intensified after the organic compounds were found to show superconductivity [3]. Over a thousand molecular conductors and over a hundred molecular superconductors have been synthesized so far. Typical molecular conductors possess non-integral oxidation states either through partial charge-transfer between a donor molecule and an acceptor molecule or through partial oxidation of a donor molecule which is a prerequisite for achieving partial filling of the conduction band. An alternative way of obtaining metallic conductance is to use a compound in which, though a number of electrons per lattice site is even, the energy gap between the highest occupied molecular orbital (HOMO) and the lowest unoccupied molecular orbital (LUMO) is small [4]. Despite great efforts, the underlying mechanisms of peculiar features observed in strongly anisotropic molecular superconductors remain unclear. Nowadays two decades after the scientific boom caused by the discovery of high temperature superconductivity, the attention of investigators is drawn again to organic superconducting systems, in particular to Fabre salts (TMTTF) $)_{2} \mathrm{X}$ (here TMTTF denotes tetrametyl-tetrathiafulvalene molecule whose structural formula is given in figure $1, \mathrm{X}$ stands for monovalent anion $\mathrm{PF}_{6}, \mathrm{AsF}_{6}, \mathrm{ClO}_{4}$ or $\mathrm{Br}$ ) and Bechgaard salts (TMTSF) $)_{2} \mathrm{X}$ (here TMTSF stands for tetrametyl-tetraselenfulvalene which differs from TMTTF by substitution of S by Se) the properties of which are believed to give the clue for solving the puzzle of mechanism of HTSC.

A few models of molecular compounds with strong electron correlations have been proposed recently [5-10] to explain the peculiarities of electrical and magnetic properties (anomalies of ther-

\footnotetext{
*E-mail: skorenky@tu.edu.te.ua
} 
<smiles>[H]</smiles>

Figure 1. The structural formula of flat tetrametyl-tetrathiafulvalene molecule.

mopower, oscillations and angular dependence of magnetoresistance, etc) of quasi-one-dimensional organic conductors. These models are extremely difficult to treat not only by the standard methods of solid state theory but also by modern strongly correlated theory methods such as Dynamical Mean Field Theory [8]. To study such models, the generalizations of the above mentioned methods were elaborated: Cellular DMFT [9], Dynamic Cluster Approximation [11] and new approaches were developed (Cluster Perturbation theory [12], Two-Particle Self Consistent Approximation [13]). Hartree-Fock approximation [10], Random Phase Approximation [14]. Bosonization and renormalization group methods $[15,16]$ have been used as well. Restrictions of standard methods are well known. The newly elaborated methods, in their turn, have their own deficiencies connected with greatly increased computation time, limitations on model parameters and difficulties with identifying the mechanisms of physical effects. At the same time, there exists a non-perturbative method $[17,18]$ which permits to explain such peculiarities of strongly correlated electron systems as metal-insulator transition [19,20], electron-hole asymmetry of ferromagnetism [21,22], conductivity [23], etc. Moreover, this method permits to take into account the correlated hopping and indirect exchange interactions, important for real strongly correlated electron systems. In this paper we formulate the model appropriate for organic superconducting systems (TMTTF) ${ }_{2} \mathrm{X}$ and $(\mathrm{TMTSF})_{2} \mathrm{X}$ and calculate the spectrum of this system using the method of work [18]. The paper is organized as follows: in section 2 we give the general formulation of the model and obtain effective Hamiltonian for the case of low electron concentration by means of canonical transformation. In section 3 we calculate the single particle energy spectrum of the model. Section 4 is devoted to the conclusions and discussion as to the applicability of the model and the model energy spectrum to real quasi-one-dimensional conductors.

\section{Effective model of quasi-one-dimensional organic conductor}

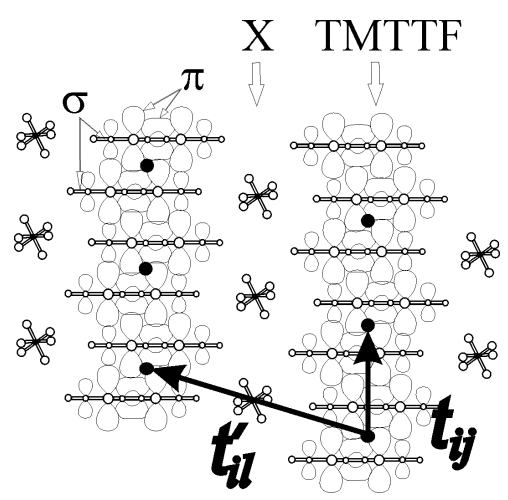

Figure 2. The structure of $(\mathrm{TMTTF})_{2} \mathrm{X}$ compound. $\pi$ and $\sigma$ orbitals are depicted. Hopping integral in the direction of a chain $t_{i j}$ and interchain hopping integral $t_{i j}^{\prime}$ are shown as well.
The crystals of organic conductors are built of zig-zag shaped stacks of flat TMTTF (TMTSF) molecules with adjoint chains of anions $\mathrm{PF}_{6}, \mathrm{AsF}_{6}, \mathrm{ClO}_{4}$ or $\mathrm{Br}$ as shown in figure 2. The chemical stability of TMTTF (TMTSF) molecule is provided by $\sigma$ - orbitals. The overlap of $\pi$-orbitals of electrons on the nearest neighbors in the chain form the conduction band in this system and provides a fairly good conductance in the direction of the chain which is about $50-300(\Omega \mathrm{cm})^{-1}$ for TMTTF and about $500(\Omega \mathrm{cm})^{-1}$ for TMTSF. Temperature dependencies of resistivity and large magnetoresistance values are similar for both Fabre and Bechgaard salts.

As a rule, TMTSF-based compounds become superconducting (SC) at very low (of the 
order of several Kelvin) temperatures under the external pressure of 10-12 kbar. The exception is $(\mathrm{TMTSF})_{2} \mathrm{ClO}_{4}$ in which $\mathrm{SC}$ state is stable at $T<1.3 \mathrm{~K}$ at atmospheric pressure. Critical pressures for TMTTF-based compounds are much higher than for their TMTSF-based counterparts. The doping of (TMTTF $)_{2} \mathrm{ClO}_{4}$ with TMTSF gives the effect similar to that with the external pressure applied. The molecular stacks are not completely independent. Therefore, interchain coupling, though small, must be taken into account. The desired Hamiltonian of the compound of (TMTSTF $)_{2} \mathrm{X}$ or $(\mathrm{TMTSF})_{2} \mathrm{X}$ type should describe the anisotropy of conductance: for $(\mathrm{TMTSF})_{2} \mathrm{X}$ with anions $\mathrm{X}=\mathrm{PF}_{6}, \mathrm{ClO}_{4}$ conductivities along crystallographic directions a:b:c are scaled as $10^{5}: 10^{3}: 10^{0}[24]$. The Fermi surface for the nearly one-dimensional band structure consists of two warped sheets at $k_{x} \approx \pm k_{\mathrm{F}}$ in the $k_{y}-k_{z}$ plane. For the compound (TMTSF) ${ }_{2} \mathrm{PF}_{6}$ the ratio of halfbandwidth parallel to the chain, $\mathrm{w}_{\|}$, to transverse halfbandwidth $\mathrm{w}_{\perp}$ deduced from the optical experiments [25] is $\approx 90: 1$ while that deduced from band structure calculations [26] is 10:1. It means that the overlap of electron wave functions along the chain is much larger than between the chains (interchain hopping in the plane is realized through anions X). Under hydrostatic pressure the ratio $\frac{w_{\|}}{w_{\perp}}$ may change. In the direction perpendicular to the plane, the hopping can be realized only if the neighboring planes are close enough (under external pressure). The overlap of molecular orbitals permitting the electrons to hop from one chain to another modifies the electron spectrum.

The family has a rich phase diagram: there we find regions of antiferromagnetic insulating state, high temperature paramagnetic insulating and metallic phases, low temperature superconducting state in which antiferromagnetic correlations can exist [27,28]. To describe such a variety of magnetic phases we have to include the direct exchange interaction and the strong intra-atomic Coulomb repulsion into the model. At strong intra-atomic Coulomb repulsion it is important to take into account the correlated hopping of electrons [29-33].

We start from the following Hamiltonian in Hubbard operators [34] reprezentation:

$$
H=H_{0}+H_{t r}+\bar{H}_{t r}+H_{t r}^{\prime}+\bar{H}^{\prime}{ }_{t r}+H_{e x}+H_{e x}^{\prime},
$$

where

$$
\begin{aligned}
H_{0}= & -\mu \sum_{i \sigma}\left(X_{i}^{\sigma}+X_{i}^{2}\right)+U \sum_{i} X_{i}^{2} \\
H_{t r}= & \sum_{\langle i j\rangle \sigma}^{\prime} t_{i j}(n) X_{i}^{\sigma 0} X_{j}^{0 \sigma}+\sum_{\langle i j\rangle \sigma}^{\prime} \tilde{t}_{i j}(n) X_{i}^{2 \sigma} X_{j}^{\sigma 2}, \\
\bar{H}_{t r}= & \sum_{\langle i j\rangle \sigma}^{\prime}\left(\bar{t}_{i j}(n)\left(X_{i}^{\downarrow 0} X_{j}^{\uparrow 2}-X_{i}^{\uparrow 0} X_{j}^{\downarrow 2}\right)+h . c .\right), \\
H_{t r}^{\prime}= & \sum_{\langle\langle i l\rangle\rangle \sigma}{ }^{\prime} t_{i l}^{\prime}(n) X_{i}^{\sigma 0} X_{l}^{0 \sigma}+\sum_{\langle\langle i l\rangle\rangle \sigma}{ }^{\prime} \tilde{t}_{i l}^{\prime}(n) X_{i}^{2 \sigma} X_{l}^{\sigma 2}, \\
\bar{H}^{\prime}{ }_{t r}= & \sum_{\langle\langle i l\rangle\rangle \sigma}{ }^{\prime}\left(\bar{t}_{i l}^{\prime}(n)\left(X_{i}^{\downarrow 0} X_{l}^{\uparrow 2}-X_{i}^{\uparrow 0} X_{l}^{\downarrow 2}\right)+h . c .\right), \\
H_{e x}= & -\frac{1}{2} \sum_{\langle i j\rangle \sigma}^{\prime} J(i j)\left(\left(X_{i}^{\sigma}+X_{i}^{2}\right)\left(X_{j}^{\sigma}+X_{j}^{2}\right)+X_{i}^{\sigma \bar{\sigma}} X_{j}^{\bar{\sigma} \sigma}\right) ; \\
H_{e x}^{\prime}= & -\frac{1}{2} \sum_{\langle\langle i l\rangle\rangle \sigma}{ }^{\prime} J(i l)\left(\left(X_{i}^{\sigma}+X_{j}^{2}\right)\left(X_{l}^{\sigma}+X_{l}^{2}\right)+X_{i}^{\sigma \bar{\sigma}} X_{l}^{\bar{\sigma} \sigma}\right) ;
\end{aligned}
$$

here $X_{i}^{k l}$ is the operator of transition of $i$ site from $|l\rangle$-state to $|k\rangle$-state (relations between electron operators and $\mathrm{X}$-operators can be found, for example, in [18]), $\mu$ is a chemical potential, $U$ is the Coulomb repulsion energy of two electrons on the same site, $\sigma=\uparrow, \downarrow$,

$$
\begin{aligned}
\tilde{t}_{i j}(n) & =t_{i j}\left(1-n \tau_{1}-2 \tau_{2}\right), \\
\bar{t}_{i j}(n) & =t_{i j}\left(1-n \tau_{1}-\tau_{2}\right) . \\
t_{i j}(n) & =t_{i j}\left(1-n \tau_{1}\right),
\end{aligned}
$$


$t_{i j}$ is the matrix element which describes the hoppings of electrons between nearest-neighbor sites of the lattice along the chain due to the electron-ion interaction (bare band hopping). Under the action of the external pressure $p$, the width $2 w$ of a band increases and the hopping parameters (as well as all inter-site processes) should be scaled [35] as $t_{i j} \rightarrow t_{i j}\left(1+\alpha u\right.$ ), where $\alpha=V_{0} / w_{0}$. $\partial w / \partial V<0$ describes the renormalization of the hopping integral at strain $u=\Delta V / V_{0} \sim p, V_{0}$ is the unit cell volume for undeformed crystal. $t_{i j}(n)$ can be identified as the hopping integral of holes, $\tilde{t}_{i j}(n)$ - as the hopping integral of doublons and $\bar{t}_{i j}(n)$ is the integral of pair creation and annihilation of holes and doublons. Analogous relations and interpretations are valid for interchain hoppings (denoted by prime). Dimensionless parameters $\tau_{1}$ and $\tau_{2}$ characterize the correlated hopping of electrons [17]. For convenience we introduce a dimensionless parameter $g=t_{i j}^{\prime} / t_{i j}$ which characterizes the anisotropy of electron hopping ( $g$ may depend on hydrostatic or chemical pressure).

In the Hamiltonian (2.1), terms (2.3)-(2.6) are of fermionic type and describe the hopping of electrons along the chain $(\langle\cdots\rangle$ denotes the summation over the nearest neighbors in the chain) and between the chains (with $\langle\langle\cdots\rangle\rangle$ denoting neighboring sites in different chains); other terms are of bosonic nature. We shall treat the fermionic and bosonic terms in different ways (the reasons are given in [18]). In the case of strong electron repulsion, when Hubbard subbands are well separated and inter-subband hopping can be neglected, we apply a canonical transformation to Hamiltonian (2.1) (this approach has a long history [36-40]) following the work [18].

$$
\tilde{H}=e^{S} H e^{-S},
$$

where

$$
S=\sum_{\langle i j\rangle}\left(L(i j)\left(X_{i}^{\uparrow 0} X_{j}^{\downarrow 2}-X_{j}^{\downarrow 0} X_{i}^{\uparrow 2}\right)-\text { h.c. }\right) .
$$

The operator $S$ is taken to exclude the processes with pair hopping of holes and doublons in the first order in hopping parameter:

$$
\bar{H}+\left[S, H_{0}\right]=0,
$$

(note that the hopping that we have just excluded was along the chain). In this way we obtain the effective exchange interaction in the chain

$$
\tilde{H}_{e x}=-\frac{1}{2} \sum_{\langle i j\rangle \sigma}^{\prime} \tilde{J}(i j)\left(X_{i}^{\sigma} X_{j}^{\bar{\sigma}}-X_{i}^{\sigma \bar{\sigma}} X_{j}^{\bar{\sigma} \sigma}\right)
$$

with the indirect exchange parameter $\tilde{J}(i j)=\left(\bar{t}_{i j}(n)\right)^{2} / U$.

The analogous procedure has to be done with Hamiltomian $\tilde{H}$ to exclude the pair hopping of holes and doublons between chains:

$$
H_{\mathrm{eff}}=e^{S^{\prime}} \tilde{H} e^{-S^{\prime}}
$$

where

$$
S^{\prime}=\sum_{\langle\langle i l\rangle\rangle}\left(L^{\prime}(i l)\left(X_{i}^{\uparrow 0} X_{l}^{\downarrow 2}-X_{l}^{\downarrow 0} X_{i}^{\uparrow 2}\right)-\text { h.c. }\right) .
$$

Finally, we obtain the effective Hamiltonian:

$$
H_{\mathrm{eff}}=H_{0}+H_{t r}+H_{t r}^{\prime}+H_{e x}+H_{e x}^{\prime}+\tilde{H}_{e x}+\tilde{H}_{e x}^{\prime}
$$

Here $\tilde{H}_{e x}^{\prime}$ is the effective interchain exchange interaction

$$
\tilde{H}_{e x}^{\prime}=-\frac{1}{2} \sum_{\langle\langle i l\rangle\rangle \sigma}^{\prime} \tilde{J}^{\prime}(i l)\left(X_{i}^{\sigma} X_{l}^{\bar{\sigma}}-X_{i}^{\sigma \bar{\sigma}} X_{l}^{\bar{\sigma} \sigma}\right)
$$

with the indirect interchain exchange parameter $\tilde{J}^{\prime}(i j)=\left(\bar{t}_{i j}^{\prime}\right)^{2} / U$. 


\section{Single-particle Green function and energy spectrum of the model}

Let us write the single-particle Green function $\left\langle\left\langle X_{s}^{0 \uparrow} \mid X_{s^{\prime}}^{\uparrow 0}\right\rangle\right\rangle$ which satisfies the equation

$$
\begin{aligned}
E\left\langle\left\langle X_{s}^{0 \uparrow} \mid X_{s^{\prime}}^{\uparrow 0}\right\rangle\right\rangle & =\frac{\delta_{s s^{\prime}}\left\langle X_{s}^{0}+X_{s}^{\uparrow}\right\rangle}{2 \pi}+\left\langle\left\langle\left[X_{s}^{0 \uparrow}, H_{\mathrm{eff}}\right] \mid X_{s^{\prime}}^{\uparrow 0}\right\rangle\right\rangle, \\
{\left[X_{s}^{0 \uparrow}, H_{t r}\right] } & =\sum_{j}^{\prime} t_{s j}(n)\left(\left(X_{s}^{0}+X_{s}^{\uparrow}\right) X_{j}^{0 \uparrow}+X_{s}^{\downarrow \uparrow} X_{j}^{\uparrow \downarrow}\right), \\
{\left[X_{s}^{0 \uparrow}, H_{t r}^{\prime}\right] } & =\sum_{r}^{\prime} t_{s r}(n)\left(\left(X_{r}^{0}+X_{s}^{\uparrow}\right) X_{r}^{0 \uparrow}+X_{s}^{\downarrow \uparrow} X_{r}^{\uparrow \downarrow}\right), \\
{\left[X_{s}^{0 \uparrow}, H_{e x}\right] } & =-\sum_{j} J(s j)\left(X_{j}^{\uparrow} X_{s}^{0 \uparrow}+X_{s}^{0 \downarrow} X_{j}^{\downarrow \uparrow}\right), \\
{\left[X_{s}^{0 \uparrow}, H_{e x}^{\prime}\right] } & =-\frac{1}{2} \sum_{r} J^{\prime}(s r)\left(X_{r}^{\uparrow} X_{s}^{0 \uparrow}+X_{s}^{0 \downarrow} X_{r}^{\downarrow \uparrow}\right), \\
{\left[X_{s}^{0 \uparrow}, \tilde{H}_{e x}\right] } & =-\sum_{j} \tilde{J}(s j)\left(X_{j}^{\downarrow} X_{s}^{0 \uparrow}-X_{s}^{0 \downarrow} X_{j}^{\downarrow \uparrow}\right), \\
{\left[X_{s}^{0 \uparrow}, \tilde{H}_{e x}^{\prime}\right] } & =-\frac{1}{2} \sum_{r} \tilde{J}^{\prime}(s r)\left(X_{r}^{\downarrow} X_{s}^{0 \uparrow}-X_{s}^{0 \downarrow} X_{r}^{\downarrow \uparrow}\right) .
\end{aligned}
$$

To obtain the closed system of equations we apply the projection procedure, proposed in works $[17,18]$. Suppose that in equation $(3.2)$

$$
\left[X_{s}^{0 \uparrow}, H_{t r}\right]=\sum_{j} \epsilon_{s}^{\uparrow}(s j) X_{j}^{0 \uparrow}, \quad\left[X_{s}^{0 \uparrow}, H_{t r}^{\prime}\right]=\sum_{r} \epsilon_{s}^{\prime \uparrow}(s r) X_{r}^{0 \uparrow},
$$

where $\epsilon_{s}^{\uparrow}(s j)$ and $\epsilon_{s}^{\prime \uparrow}(s r)$ are non-operator expressions which we calculate using the method of work [18]. This approach gives a correct description of metal-insulator transition [20] in the Hubbard model (in particular, the calculated concentration of polar states is in accordance with the results of DMFT [8]) and reproduces the exact results [41,33] found for the partial case of the model with correlated hopping of electrons. For the exchange terms we apply the mean-field approximation:

$$
\begin{array}{ll}
{\left[X_{s}^{0 \uparrow}, H_{e x}\right]=-z_{1} J \lambda_{s}^{\uparrow} X_{s}^{0 \uparrow},} & {\left[X_{s}^{0 \uparrow}, H_{e x}^{\prime}\right]=-\frac{z_{2} J^{\prime}}{2} \lambda_{s}^{\prime \uparrow} X_{s}^{0 \uparrow},} \\
{\left[X_{s}^{0 \uparrow}, \tilde{H}_{e x}\right]=-z_{1} \tilde{J} \tilde{\lambda}_{s}^{\uparrow} X_{s}^{0 \uparrow},} & {\left[X_{s}^{0 \uparrow}, \tilde{H}_{e x}^{\prime}\right]=-\frac{z_{2} \tilde{J}^{\prime}}{2} \tilde{\lambda}_{s}^{\prime \uparrow} X_{s}^{0 \uparrow} .}
\end{array}
$$

Here $z_{1}$ is the number of the nearest neighbors in the chain, $z_{2}$ is the number of neighboring sites belonging to other chains.

Analogous procedure is performed for the Green function $\left\langle\left\langle X_{p}^{0 \uparrow} \mid X_{s^{\prime}}^{\uparrow 0}\right\rangle\right\rangle$.

After the Fourier transformation, the system of equations has the following form

$$
\begin{aligned}
& \left(E+\mu-\alpha_{s}^{\uparrow} t_{\mathbf{k}}(n)-\beta_{s}^{\uparrow}(\mathbf{k})+C_{s}^{\uparrow}\right)\left\langle\left\langle X_{s}^{0 \uparrow} \mid X_{s^{\prime}}^{\uparrow 0}\right\rangle\right\rangle_{\mathbf{k}}-\alpha_{s}^{\prime \uparrow} t_{\mathbf{k}}^{\prime}(n)\left\langle\left\langle X_{p}^{0 \uparrow} \mid X_{s^{\prime}}^{\uparrow 0}\right\rangle\right\rangle_{\mathbf{k}}=\frac{\left\langle X_{s}^{0}+X_{s}^{\uparrow}\right\rangle}{2 \pi}(3.10) \\
& \left(E+\mu-\alpha_{p}^{\uparrow} t_{\mathbf{k}}(n)-\beta_{p}^{\uparrow}(\mathbf{k})+C_{p}^{\uparrow}\right)\left\langle\left\langle X_{p}^{0 \uparrow} \mid X_{s^{\prime}}^{\uparrow 0}\right\rangle\right\rangle_{\mathbf{k}}-\alpha_{p}^{\prime \uparrow} t_{\mathbf{k}}^{\prime}(n)\left\langle\left\langle X_{s}^{0 \uparrow} \mid X_{s^{\prime}}^{\uparrow 0}\right\rangle_{\mathbf{k}}=0,\right.
\end{aligned}
$$

where $\epsilon_{x}^{\uparrow}(\mathbf{k})=\alpha_{x}^{\uparrow} t_{\mathbf{k}}(n)+\beta_{x}^{\uparrow}(\mathbf{k}), \epsilon_{x}^{\prime \uparrow}(\mathbf{k})=\alpha_{x}^{\prime \uparrow} t_{\mathbf{k}}^{\prime}(n)$ and notation $C_{x}^{\uparrow}=z_{1} J \lambda_{x}^{\uparrow}+1 / 2 z_{2} J^{\prime} \lambda_{x}^{\prime \uparrow}+z_{1} \tilde{J} \tilde{\lambda}_{x}^{\uparrow}+$ $1 / 2 z_{2} \tilde{J}^{\prime} \tilde{\lambda}_{x}^{\prime}$ is introduced. 
We obtain the solution of the system of equations (3.10):

$$
\begin{aligned}
& \left\langle\left\langle X_{s}^{0 \uparrow} \mid X_{s^{\prime}}^{\uparrow 0}\right\rangle\right\rangle_{\mathbf{k}}=\frac{\left\langle X_{s}^{0}+X_{s}^{\uparrow}\right\rangle}{2 \pi}\left(\frac{A_{\mathbf{k}}}{E-E_{1}^{\uparrow}(\mathbf{k})}+\frac{B_{\mathbf{k}}}{E-E_{2}^{\uparrow}(\mathbf{k})}\right), \\
& A_{\mathbf{k}}=\frac{1}{2}\left(1-\frac{K^{\uparrow}}{\sqrt{\left(K^{\uparrow}\right)^{2}+4 \alpha_{s}^{\prime \uparrow} \alpha_{p}^{\prime \uparrow}\left(t_{\mathbf{k}}^{\prime}\right)^{2}}}\right), \quad B_{\mathbf{k}}=1-A_{\mathbf{k}} .
\end{aligned}
$$

Here the energy spectrum is

$$
E_{1,2}^{\uparrow}(\mathbf{k})=-\mu+\frac{1}{2} \sum_{x}\left(\alpha_{x}^{\uparrow} t_{\mathbf{k}}(n)+\beta_{x}^{\uparrow}-C_{x}^{\uparrow}\right) \mp \frac{1}{2} \sqrt{\left(K^{\uparrow}\right)^{2}+4 \alpha_{s}^{\prime \uparrow} \alpha_{p}^{\prime \uparrow}\left(t_{\mathbf{k}}^{\prime}\right)^{2}},
$$

where $K^{\uparrow}=\left(\alpha_{s}^{\uparrow}-\alpha_{p}^{\uparrow}\right) t_{\mathbf{k}}(n)+\beta_{s}^{\uparrow}-\beta_{p}^{\uparrow}-\left(C_{s}^{\uparrow}-C_{p}^{\uparrow}\right)$. The approximate procedure of square root expansion in equation (3.12) in parameter $g^{2}=\left(t_{\mathbf{k}}^{\prime} / t_{\mathbf{k}}\right)^{2} \ll 1$ permits to identify the quantities $\alpha_{x}^{\sigma}$ and $\alpha_{x}^{\prime \sigma}$ as correlation narrowing factors and $\beta_{x}^{\sigma}(\mathbf{k})$ as spin-dependent shifts of subband centers. The values of correlation narrowing factors can be found by making use of quasiclassical approximation for calculation of the averages of $\left\langle X_{s}^{\downarrow \uparrow} X_{j}^{\uparrow \downarrow}\right\rangle$ type with the choice of expressions for $\left\langle X_{s}^{\sigma}\right\rangle$ appropriate to a magnetic state under consideration. As an example, we give the values obtained for paramagnetic state:

$$
\alpha_{i}^{\sigma}=\alpha_{i}^{\prime \sigma}=\frac{(2-n)^{2}+n^{2}}{2(2-n)}, \quad \beta_{i}^{\sigma}=\frac{w_{\|}(n)(1-n) n}{2-n},
$$

ferromagnetic state:

$$
\alpha_{i}^{\sigma}=\alpha_{i}^{\prime \sigma}=\frac{\left(2-n+\eta_{\sigma} m\right)^{2}+n^{2}-m^{2}}{\left(2-n+\eta_{\sigma} m\right)}, \quad \beta_{i}^{\sigma}=\frac{w_{\|}(n)(1-n)\left(n-\eta_{\sigma} m\right)}{2-n+\eta_{\sigma} m},
$$

and antiferromagnetic state:

$$
\alpha_{i}^{\sigma}=\alpha_{i}^{\prime \sigma}=\frac{\left(2-n+\eta_{\sigma} \eta_{i} m\right)^{2}+n^{2}-m^{2}}{\left(2-n+\eta_{\sigma} \eta_{i} m\right)}, \quad \beta_{i}^{\sigma}=\frac{w_{\|}(n)(1-n)\left(n-\eta_{\sigma} \eta_{i} m\right)}{2-n+\eta_{\sigma} \eta_{i} m},
$$

where $\eta_{\sigma}=1$ if $\sigma=\uparrow$ and -1 otherwise, $\eta_{i}=1$ if $i=s$ and $\eta_{i}=-1$ if $i=p$. In the above expressions $w_{\| \mid}(n)=z_{1}\left|t_{i j}(n)\right|, n$ denotes the electron concentration and $m$ is the magnetization. In a similar way the values $\lambda_{i}^{\sigma}, \lambda_{i}^{\prime \sigma}, \tilde{\lambda}_{i}^{\sigma}$ and $\tilde{\lambda}_{i}^{\prime \sigma}$ can be calculated.

As one can see from $\mathbf{k}$-dependencies of the spectrum (3.12), in paramagnetic state we have single correlation-narrowed band with shifted chemical potential, in ferromagnetic state spin-splitting of the band takes place, in antiferromagnetic state the splitting is fourfold due to the combined effect of transversal hopping and antiferromagnetic sublattice formation. The instabilities (induced by transversal hopping) of fermi-liquid described by energy spectrum (3.12) may occur when the energy minimum corresponds to the states of electrons with $\mathbf{k} \neq \mathbf{0}$ in the first Brillouin zone. An effective inter-stack exchange can result after the onset of long-range order. The dimerization, found in (TMTSF $)_{2} \mathrm{PF}_{6}$ at $30 \mathrm{~K}$, can be reflected by the change of the number of interchain (NN) neighbors $z_{2}$ from 2 in antiferromagnetic state to 4 in the dimerized state. The effective mass, calculated using the energy spectrum (3.12) is strongly anisotropic [45], in agreement with the experimental findings. If we neglect the correlated hopping in the model described by Hamiltonian (2.1), we obtain the model similar to that considered in works [14,15], then the spectrum (3.12) takes the form similar to the corresponding results of works $[15,42,43]$ in the high-temperature paramagnetic state and of works $[5,44]$ in the high-temperature antiferromagnetic state.

To summarize, we have formulated the effective model of quasi-one-dimensional molecular conductor with strong intra-atomic Coulomb interaction and the correlated hopping of electrons. In this case the most convenient representation is the $X$-operator representation. To calculate the energy spectrum, the non-perturbative projection procedure $[17,18]$ was used. The energy spectrum (3.12) can help to explain the phase transitions in (TMTTF) $)_{2} \mathrm{X}$ and (TMTSF) ${ }_{2} \mathrm{X}$ salts when either hydrostatic or chemical pressure ( $\mathrm{S} / \mathrm{Se}$ atom substitution or anion $\mathrm{X}=\mathrm{PF}_{6}, \mathrm{AsF}_{6}, \mathrm{Br}$ substitution) is applied or temperature changes. 


\section{Acknowlegements}

This work was supported by Ukrainian Fund for Fundamental Research under grant No. GP/F8/44.

\section{References}

1. Akamatu H., et al, Nature, 1954, 173, 168.

2. Bechgaard K., Jerome D., Uspiekhi Fiz. Nauk, 1983, 140, 671 (in Russian).

3. Jerome D., et al, J. Phys. Lett., 1980, 41, 95.

4. Tanaka H., et al, Science, 2001, 291, 285-288.

5. Kino H., Fukuyama H., J. Phys. Soc. Jpn., 1981, 46, 1142.

6. McKenzie R., Comments Cond. Matt. Phys., 1998, 18, 309.

7. Kuroki K., Aoki H., Phys. Rev. B, 1999, 60, 3060.

8. Georges A., et al, Rev. Mod. Phys., 1996, 68, 13.

9. Parcollet O., Biroli G., Kotliar G., Phys. Rev. Lett., 2004, 92, 226402.

10. Hofstetter W., Vollhardt D., Ann. Physik, 1998, 7, 48.

11. Hettler M.H., et al, Phys. Rev. B, 2000, 61, 12739.

12. Senechal D., Perez D., Pioro-Ladriere M., Phys. Rev. Lett., 2000, 84, 522.

13. Allen S., Tremblay A.M.-S.T., Vilk Y.M. Theoretical Methods for Strongly Correlated Electrons, eds. Senechal D., et al. CRM Series in Mathematical Physics. Springer, New York, 2003.

14. Yamaji K., Journ. Phys. Soc. Jpn., 1983, 52, 1361.

15. Bourbonnais C. Trends in High Magnetic Fields Science, eds. C. Berthier, et al. Springer, New York, 2002.

16. Louis K., Alvarez J.V., Gros C., Phys. Rev. B, 2001, 64, 113106.

17. Didukh L., Journ. of Phys. Stud., 1997, 1, 241 (in Ukrainian).

18. Didukh L., Condens. Matter Phys., 1998, 1, 125.

19. Didukh L., Hankevych V., Dovhopyaty Yu., Physica B, 1999, 259-261, 719.

20. Didukh L., Skorenkyy Yu., Condens. Matt. Phys., 2000, 3, 787.

21. Didukh L., Kramar O., Skorenkyy Yu., Acta Phys. Pol. B, 2003, 34, 791.

22. Didukh L., et al, Phys. Rev. B, 2001, 64, 144428.

23. Didukh L., et al. Preprint of Institute for Condensed Matter Physics, ICMP-03-31U, 30 p. Lviv, 2003, (in Ukrainian).

24. Scheven U.M., et al, Phys. Rev. B, 1997, 56, 7804.

25. Jakobsen C.S., Tanner D.B., Bechgaarg K., Phys. Rev. Lett., 1981, 46, 1142.

26. Grant P.M., Phys. Rev. B, 1981, 26, 6888.

27. Ishiguiro T., Yamaji K., Saito G. Organic Superconductors. Springer, Berlin, 1998.

28. Jaccard D., et al, J. Phys.: Condens. Matter, 2001, 13, L89.

29. Didukh L.D., Fiz. Tverdogo Tela, 1977, 19, 1217 (in Russian).

30. Didukh L., Preprint of Institute for Condensed Matter Physics, ICMP-92-9P, 32 p., Lviv, 1992, (in Russian).

31. Hirsch J.E., Marsiglio F., Phys. Rev. B, 1989, 39, 11515; Hirsch J.E., Physica B, 1994, 199-200, 366.

32. Lin H.Q., Hirsch J.E., Phys. Rev. B, 1995, 52, 16155; Amadon J.C., Hirsch J.E., Phys. Rev. B, 1996, $\mathbf{5 4}, 6364$.

33. Gagliano E.R., et al, Phys. Rev. B, 1995, 51, 14012; Arrachea L., et al, Phys. Rev. B, 1997, 55, 1173.

34. Hubbard J., Proc. Roy. Soc. A, 1965, 285, 542.

35. Grygorchuk R.A., Stasyuk I.V., Ukr. Phys. Journ., 1980, 25, 404 (in Russian).

36. Bogolyubov M.M. Lectures on quantum statistics. Radianska shkola, Kyiv, 1948 (in Ukrainian).

37. Harris A.B., Lange V., Phys. Rev., 1967, 157, 295.

38. Didukh L.D., Stasyuk I.V., Fiz. Met. Metalov., 1968, 26, 582 (in Russian); Ukr. Fiz. Zhurn., 1968, 13, 899 (in Russian).

39. Chao K.A., Spalek J., Oles A., Phys. Rev. B, 1978, 18, 3453.

40. Gros C., Joynt R., Rice T.M., Phys. Rev. B, 1987, 36, 381.

41. Strack R., Vollhardt D., Phys. Rev. Lett., 1993, 70, 2637.

42. Maki K., Phys. Rev. B, 1993, 47, 11506.

43. Danner G.M., Chaikin P.M., Hannahs S.T. Phys. Rev. B, 1996, 53, 2727.

44. Lebed A.G., Phys. Rev. B, 1997, 55, 1299.

45. Skorenkyy Yu., O.Kramar, in preparaton. 


\title{
Енергетичний спектр органічних квазіодновимірних провідників з перестрибуванням електронів між вузлами, наступними за найближчими та корельованим переносом
}

\author{
Ю.Скоренький, О.Крамар
}

Тернопільський державний технічний університет

ім. І. Пулюя, кафедра фізики,

Україна, 46001 Тернопіль, вул. Руська, 56

Отримано 3 серпня 2005 р., в остаточному вигляді - 27 лютого 2006 р.

Розглядається модель органічних одновимірних провідників (TMTTF) ${ }_{2} \mathrm{X}$ i (TMTSF) ${ }_{2} \mathrm{X}$. Анізотропний характер цих сполук моделюється двома різними параметрами перестрибування електронів: $t$ між найближчими сусідами в ланцюжку молекул тетраметил-тетратіафулвалену (TMTTF) чи тетраметилтетраселенфулвалену (TMTSF) та $t^{\prime}$ між ланцюжками. Врахування корельованого переносу дозволяє описати вплив заселеності вузлів на процеси перестрибування електронів. В режимі сильної кулонівської взаємодії високоенергетичні процеси виключаються послідовним застосуванням двох канонічних перетворень. Отримано ефективний гамільтоніан для концентрації електронів $n<1$, який містить кінетичний надобмін антиферомагнітної природи. 3 іншого боку, міжланцюжковий перенос та корельований перенос дестабілізують антиферомагнітне впорядкування. Розраховано енергетичний спектр моделі, обговорено його застосування до квазіодновимірних провідників.

Ключові слова: квазіодновимірні органічні провідники, міжланцюжковий перенос, корельований перенос, непряма обмінна взаємодія

PACS: $74.25 .-q, 71.10 . F d$ 